\title{
Pattern competition and homoclinic crossing
}

\author{
Yu Tan and Jian-min Mao \\ Department of Mathematics, Hong Kong University of Science and Technology, Clearwater Bay, Kowloon, Hong Kong
}

(Received 21 May 1997; revised manuscript received 17 September 1997)

\begin{abstract}
We study the mechanism of pattern competition in a system described by the generalized nonlinear Schrödinger (GNS) equation with periodic boundary conditions. We achieve pattern selection and pattern competition by applying artificial disturbances in a numerical simulation and observe a correspondence between pattern competition and homoclinic crossing. This observation is further confirmed by a perturbation analysis in the neighborhood of the steady-state solution of the GNS equation near the threshold of modulation instability. The analysis gives an equation for an "order function"' based on which we show that the homoclinic structure exists in the system and that homoclinic crossings are the mechanism for pattern competition. [S1063-651X(98)09701-3]
\end{abstract}

PACS number(s): 05.45.+b, 52.35.Sb

\section{INTRODUCTION}

Spatially extended dynamical systems represent many physical situations in the fields of hydrodynamics, plasma physics, chemistry, biology, etc. Spatial-temporal chaos and space-presented coherence with time-presented chaos are of particular interest [1-4]. Many numerical simulations for different systems have provided rich information on chaotic behavior. Some perturbation analyses have also been performed $[5,6]$. It has been found that formation and competition of spatial coherent structures (patterns) play important roles in the dynamics of the systems $[3,4,7]$. In spite of remarkable progress in pattern dynamics, some fundamental questions are still unanswered. One of them concerns the onset of pattern competition: what is the mechanism of pattern competition?

To gain insight into this question, we take the following one-dimensional generalized nonlinear Schrödinger (GNS) equation as a model,

$$
i \partial_{t} E+\partial_{x}^{2} E+\left(|E|^{2}-g|E|^{4}\right) E=0, \quad 0 \leqslant x \leqslant L, \quad t>0,
$$

where $E(x, t)$ is the wave function and $g$ is a small parameter. This equation arises in such diversified physical contexts as plasma physics, nonlinear optics, water wave theory, vortex filament dynamics, etc. [2,3]. Numerical studies have discovered that the system has a homoclinic structure [8] and that there is a thin disorder layer in the neighborhood of the homoclinic orbit [9].

In this study, the equation serves as a model for nearly integrable Hamiltonian systems that exhibit complex spatialtemporal phenomena: chaos in time series and competition between patterns. We study this equation with the periodic boundary condition $E(x=0, t)=E(x=L, t)$. We first select patterns and achieve pattern competition in a numerical simulation by properly choosing initial conditions that are artificial disturbances added to the spatially homogeneous steady state. In the simulation we observe a correspondence between pattern competition and homoclinic crossing. This correspondence is further confirmed by a perturbation analysis in the neighborhood of the steady-state solution of the
GNS equation near the threshold of modulation instability. The analysis results in an equation for an "order function", of time, $\alpha(t)$, which controls evolution of the system. From this analytical result, we show that the system has a homoclinic structure and that the mechanism of pattern competition is actually the homoclinic crossings.

This paper is organized as follows. Section II includes the numerical simulation. In Sec. III, we present the perturbation analysis. Results of the analysis are used in Sec. IV to reveal the mechanism of pattern competition. Conclusions are drawn in Sec. V.

\section{PATTERN SELECTION AND PATTERN COMPETITION}

In this section, we numerically study the GNS equation (1) with the same periodic boundary condition but with different initial conditions. Pattern selection and pattern competition are induced by properly chosen artificial disturbances to the steady-state solution of the GNS equation. Results of the simulation suggest that there is a correspondence between pattern competition and homoclinic crossing.

Equation (1) is equivalent to

$$
\begin{array}{r}
i \partial_{t} E+\partial_{x}^{2} E+\left(|E|^{2}-g|E|^{4}-\left|E_{0}\right|^{2}+g\left|E_{0}\right|^{4}\right) E=0, \\
0 \leqslant x \leqslant L, \quad t>0,
\end{array}
$$

where $E_{0}=\sqrt{N}$ is a constant. Here $N=\int|E|^{2} d x$ is the total number of quasi-particles, a conserved quantity. This equation is equivalent to Eq. (1) because the constant terms in Eq. (2), $-\left|E_{0}\right|^{2}+g\left|E_{0}\right|^{4}$, can be moved by the trivial gauge transformation, $\quad E(x, t) \rightarrow E(x, t) e^{i\left(\left|E_{0}\right|^{2}-g\left|E_{0}\right|^{4}\right) t}$. Consequently, we will work on Eq. (2) instead of Eq. (1).

Obviously, the equation has a steady-state solution,

$$
E(x, t)=E_{0} .
$$

It is easy to show by linearization that this solution is stable when $L<L_{0}$, where $L_{0}=\sqrt{2} \pi /\left(E_{0} \sqrt{1-2 g E_{0}^{2}}\right)$ is the critical value of the system's length, and is unstable when $L>L_{0}$. 
To gain insight into how to choose proper artificial disturbances to the steady-state solution so that a desirable pattern will be selected, we first perform linearization near this spatially homogeneous steady state.

\section{A. Linearization}

Near the steady state $E(x, t)=E_{0}$, the linearized equation is

$$
\partial_{t} \delta E=A \delta E,
$$

where

$$
\delta E=\left(\begin{array}{l}
\delta u \\
\delta v
\end{array}\right), \quad A=\left(\begin{array}{cc}
0 & -\partial_{x}^{2} \\
\partial_{x}^{2}+k_{0}^{2} & 0
\end{array}\right) .
$$

Here $\delta u$ is the real part of $\delta E, \delta v$ is the image part, and

$$
k_{0}=\sqrt{2 E_{0}^{2}\left(1-2 g E_{0}^{2}\right)} .
$$

The linearized equation (4) has the solution

$$
\delta E=\left(c_{1} e^{-\lambda t} X_{1}+c_{2} e^{\lambda t} X_{2}\right) \cos k x,
$$

where $c_{1}$ and $c_{2}$ are constants, $k$ is the wave number, $\pm \lambda$ are the eigenvalues,

$$
\lambda=k^{2} \gamma, \quad \gamma=\sqrt{\left(k_{0} / k\right)^{2}-1},
$$

and $X_{1}$ and $X_{2}$ are the eigenvectors respectively corresponding to $\lambda$ and $-\lambda$,

$$
X_{1}=\left(\begin{array}{c}
1 \\
-\gamma
\end{array}\right), \quad X_{2}=\left(\begin{array}{l}
1 \\
\gamma
\end{array}\right) .
$$

It is easy to see that $\lambda=0$ when $k=k_{0}$. So $k_{0}$ is the critical value of the wave number for the modulation instability.

\section{B. Numerical simulation}

Setting $t=0$ in Eq. (6), the initial deviation from the steady state is $\delta E=\left(c_{1} X_{1}+c_{2} X_{2}\right) \cos (k x)$. This motivates us to take the initial condition for the GNS equation (2) as

$$
E(x, t=0)=E_{0}+\epsilon E_{0}\left(c_{1} X_{1}+c_{2} X_{2}\right) \cos (k x),
$$

where $\epsilon$ is a small parameter for the disturbance. That is, the initial condition is the steady state with a small artificial disturbance. There are five possible cases for this form of artificial disturbance depending on the signs of $c_{1}$ and $c_{2}$ : (i) $c_{1}<0$ and $c_{2}>0$, (ii) $c_{1}>0$ and $c_{2}<0$, (iii) $c_{1}>0$ and $c_{2}$ $>0$, (iv) $c_{1}<0$ and $c_{2}<0$, and (v) $c_{1}=0$ but $c_{2} \neq 0$, or $c_{2}$ $=0$ but $c_{1} \neq 0$. Actually, case (v) corresponds to the homoclinic orbit.

Starting from an initial condition in the form of Eq. (9) with $c_{1}$ and $c_{2}$ specified, we integrate Eq. (2) by using the improved split method of the third order. In the simulation we set $E_{0}=2, g=0.05$ and $L=1.4 L_{0}$ (so $k<k_{0}$ ) in Eq. (2), and $\epsilon=0.1$ and $k=0.8 k_{0}$ in Eq. (9).
For case (i) with $c_{1}=-1.0$ and $c_{2}=1.0$, the result of the simulation is shown in Fig. 1(a). We plot $\rho(x, t)$, which is defined by $E(x, t)=\sqrt{\rho(x, t)} e^{i \theta(x, t)}$ and is called the "squareroot" amplitude $[\theta(x, t)$ is the phase function]. It can be seen from the figure that a "caviton" in the middle of the space $(x=L / 2)$ disappears and reappears periodically over time. This pattern will be called pattern type I. Figure 1(b) is for case (ii) with $c_{1}=1.0, c_{2}=-1.0$. In the figure, a "soliton" disappears and reappears periodically at $x=L / 2$. This pattern will be called type II. The periodic appearance of the caviton or soliton is due to the Fermi-Pasta-Ulam recurrence [10].

Figures 2(a) and 2(b) are for case (iii) with $c_{1}=1.0$ and $c_{2}=1.0$, and for case (iv) with $c_{1}=-1.0$ and $c_{2}=-1.0$, respectively. We observe a regular composition of pattern types I and II. Patterns seen in Figs. 2(a) and 2(b) are the same except with a phase shift.

Figure 3(a) is obtained by setting $c_{1}=1.0, c_{2}=-0.4$ in Eq. (9). This initial condition is closer to the homoclinic orbit than the other cases studied above. As shown in the figure, pattern types I and II appear randomly: no single pattern dominates another and it is impossible to predict what pattern type will emerge in the next moment. We thus say that there is a pattern competition.

Figures 1(c), 2(c), and 3(b) are plots in phase space, i.e., $\rho(0, t)$ versus $(d / d t) \rho(0, t)$. In Fig. 1(c), if the initial condition is at a point on the right island [for case (i)], then the motion will stay on the right island. On the other hand, if motion starts at a point on the left island [for case (ii)], then it will remain on the island. In Fig. 2(c), we start at a point on an enclosing invariant torus and the motion will stay on the torus. If the starting point is on the upper (lower) part of the torus, then we have case (iii) [case (iv)]. For all these cases (i)-(iv), there are no homoclinic crossings nor pattern competition. In Fig. 3(b), however, we start at a point on the disorder layer around the homoclinic orbit, homoclinic crossings occur and pattern competition is achieved. This suggests that there may be a correspondence between pattern competition and homoclinic crossing: if there is a pattern competition, then there are homoclinic crossings (Fig. 3); otherwise, there is no homoclinic crossing (Figs. 1 and 2). This correspondence will be confirmed by an analysis presented in the following sections.

\section{PERTURBATION ANALYSIS}

In this section, we present a perturbation analysis for the system described by the GNS equation (2) with the periodic boundary condition. The initial condition will not be explicitly given in the analysis. Instead, a function, $\alpha(t)$, which depends on the initial condition, is introduced. An equation for $\alpha(t)$ is obtained as a result of the analysis. The approximate solution given by the perturbation analysis agrees well with the numerically determined exact solution, as shown in Fig. 4.

The perturbation analysis is performed in the neighborhood of the steady-state solution of the GNS equation (2) and near the threshold of modulation instability. We consider the problem in the extended state space $(\rho, \theta, k, \omega)$, where $\rho$ and $\theta$ are the square-root amplitude and the phase function of the wave function as defined previously, $E(x, t)$ $=\sqrt{\rho(x, t)} e^{i \theta(x, t)}, k=2 \pi / L$ is the wave number and $\omega$ is the fundamental frequency of the system. Hence our perturbation analysis is in the neighborhood of the following point in the extended state space, 


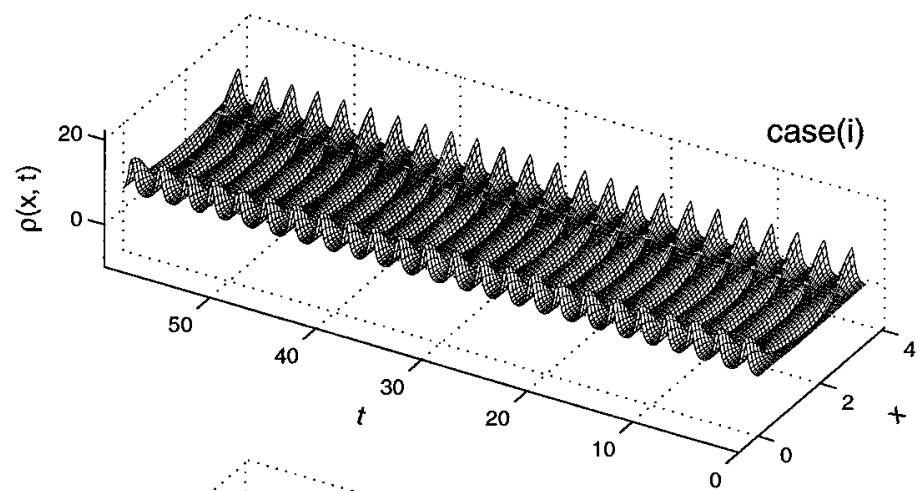

(a)

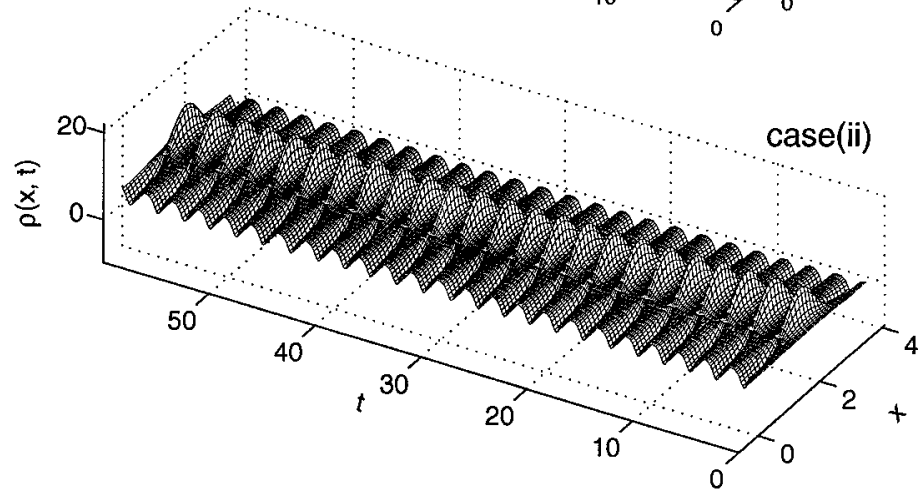

(b)

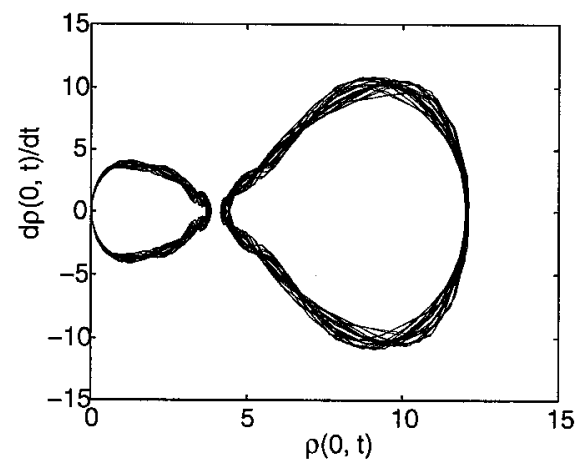

(c)

FIG. 1. The square-root amplitude function $\rho(x, t)=|E(x, t)|^{2}$ given by the numerical simulation (a) for case (i) and (b) for case (ii) of the initial condition in the form of Eq. (9). The pattern exhibited in (a) is called type I and pattern in (b) called type II. Note that there is no pattern competition. (c) Homoclinic structure in the phase space $[\rho(0, t)$ vs $d \rho(0, t) / d t]$. Note that there is no homoclinic crossing.

$$
\left(\rho_{0}, \theta_{0}, k_{0}, \omega_{0}\right)=\left[\left|E_{0}\right|^{2}, 0, \sqrt{2\left|E_{0}\right|^{2}\left(1-2 g\left|E_{0}\right|^{2}\right)}, 0\right] \text {, }
$$

where $(\rho, \theta)=\left(\rho_{0}, \theta_{0}\right)$ is the steady-state solution given in Eq. (3), $k=k_{0}$ is the critical wave number for the modulation instability given in Eq. (5), and $\omega=\omega_{0}=0$ is the frequency for the steady-state on the threshold of modulation instability. In the neighborhood of $\left(\rho_{0}, \theta_{0}, k_{0}, \omega_{0}\right)$, the variables can be expanded in series, i.e.,

$$
\begin{gathered}
\rho=\rho_{0}+\epsilon \rho_{1}+\epsilon^{2} \rho_{2}+\epsilon^{3} \rho_{3}+\cdots, \\
\theta=\epsilon \theta_{1}+\epsilon^{2} \theta_{2}+\epsilon^{3} \theta_{3}+\cdots, \\
k=k_{0}+\epsilon k_{1}+\epsilon^{2} k_{2}+\epsilon^{3} k_{3}+\cdots, \\
\omega=\epsilon \omega_{1}+\epsilon^{2} \omega_{2}+\epsilon^{3} \omega_{3}+\cdots,
\end{gathered}
$$

where $\epsilon$ is the perturbation parameter and will be set to 1 in the final stage. We have performed the perturbation analysis and found that $\omega_{i}$ is involved only in the ratio $\omega_{i} / \omega_{1}$ for $i$ $=1,2, \ldots$. For the sake of simplicity, we therefore introduce the scaled frequency $\omega^{\prime}=\omega / \omega_{1}=\epsilon\left(1+\epsilon \omega_{2}^{\prime}+\epsilon^{2} \omega_{3}^{\prime}\right.$ $+\cdots)$, where $\omega_{i}^{\prime}=\omega_{i} / \omega_{1}$ for $i=2,3, \ldots$.

Equations for $\rho(x, t)$ and $\theta(x, t)$ are, from the GNS equation (2),

$$
\begin{aligned}
k^{2}\left[\partial_{x}^{2} \rho-\right. & \left.\frac{\left(\partial_{x} \rho\right)^{2}}{2 \rho}-2 \rho\left(\partial_{x} \theta\right)^{2}\right] \\
= & \omega^{\prime} 2 \rho \partial_{t} \theta-\left(\rho-g \rho^{2}-\rho_{0}+g \rho_{0}^{2}\right) 2 \rho, \\
k^{2}\left[\partial_{x}^{2} \theta+\frac{\left(\partial_{x} \theta\right)\left(\partial_{x} \rho\right)}{\rho}\right] & =-\omega^{\prime} \frac{1}{2 \rho} \partial_{t} \rho .
\end{aligned}
$$

Here we have changed the space and time variables by $x \rightarrow x^{\prime}=k x$ and $t \rightarrow t^{\prime}=\omega^{\prime} t$. We have assumed that these changes have been made and have dropped the primes on the variables. 


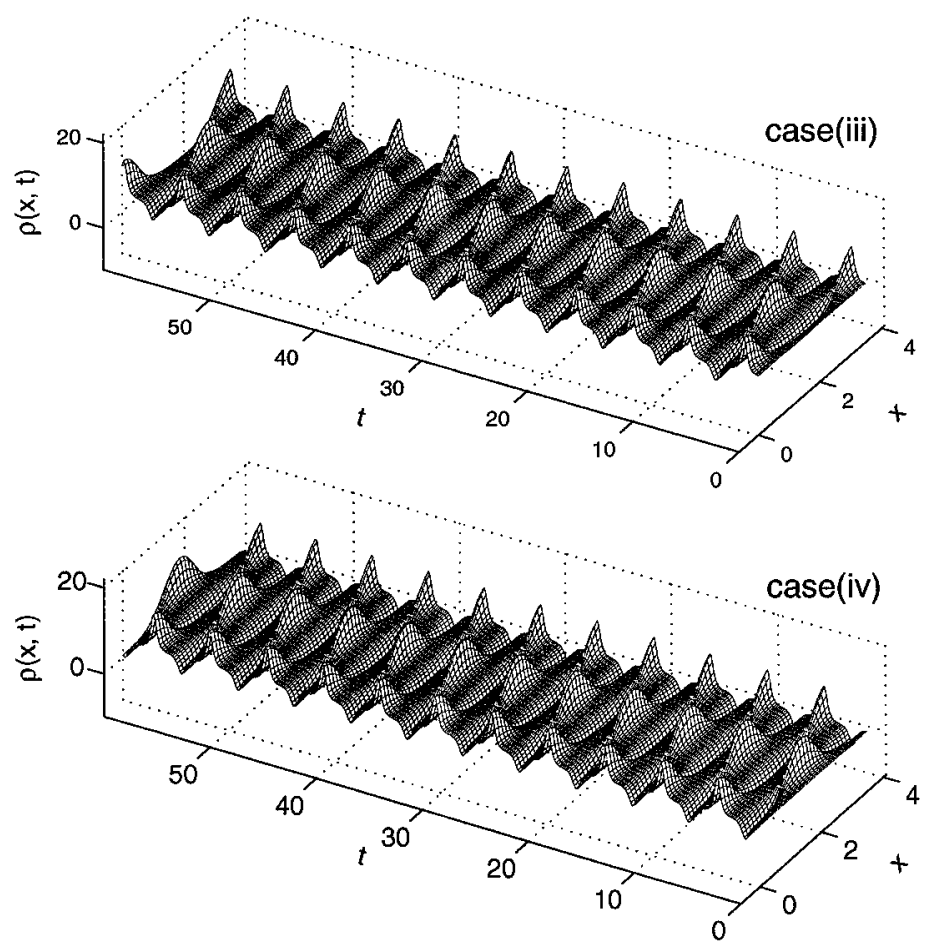

(a)

(b)

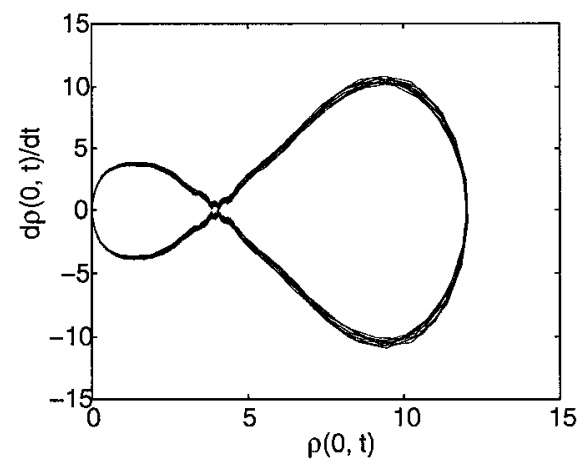

(c)

FIG. 2. Similar to Fig. 1 but (a) for case (iii) and (b) for case (iv). The patterns for the two cases are identical, except with a phase shift. They are a regular combination of pattern types I and II. Note that there is no pattern competition. (c) Homoclinic structure in the phase space. Note that there is no homoclinic crossing

Substituting Eq. (11) into Eq. (12), we obtain two equations for each order in $\epsilon$. To the zeroth order in $\epsilon$, Eqs. (12) gives the steady-state solution. For the first order in $\epsilon$, one gets

$$
\begin{gathered}
\partial_{x}^{2} \rho_{1}+\rho_{1}=0, \\
\partial_{x}^{2} \theta_{1}=0 .
\end{gathered}
$$

These equations have solutions $\rho_{1}=\alpha(t) \cos [x+\phi(t)]$ and $\theta_{1}=\beta(t)+x \sigma(t)$, where $\alpha(t), \beta(t), \phi(t)$, and $\sigma(t)$ depend on the initial conditions. In this study, we are interested only in the case where the system has spatial reflection symmetry about $x=0$. Therefore the solutions for the first order in $\epsilon$ are

$$
\begin{gathered}
\rho_{1}=\alpha(t) \cos (x), \\
\theta_{1}=\beta(t) .
\end{gathered}
$$

For the second order in $\epsilon$, we have

$$
\begin{aligned}
\partial_{x}^{2} \rho_{2}+\rho_{2}= & \frac{1}{1-2 g \rho_{0}}\left[\partial_{t} \beta+\left(g-\frac{1}{4 \rho_{0}}\right) \alpha^{2}\right] \\
& +\frac{2 k_{1}}{\sqrt{2 \rho_{0}\left(1-2 g \rho_{0}\right)}} \alpha \cos (x) \\
& +\frac{1}{1-2 g \rho_{0}}\left[2 g-\frac{3}{4 \rho_{0}}\right] \alpha^{2} \cos (2 x), \\
\partial_{x}^{2} \theta_{2} & =-\frac{1}{2 \rho_{0} k_{0}^{2}} \partial_{t} \alpha(t) \cos (x) .
\end{aligned}
$$

The coefficient of $\cos (x)$ in the first equation has to be zero (that is, $k_{1}=0$ ) for its solution to satisfy the periodic boundary condition. We must also set the background terms in the first equation to zero in order to satisfy the conservation of quasiparticles, i.e., $\partial_{t} \beta+\left(g-1 / 4 \rho_{0}\right) \alpha^{2}=0$, or 


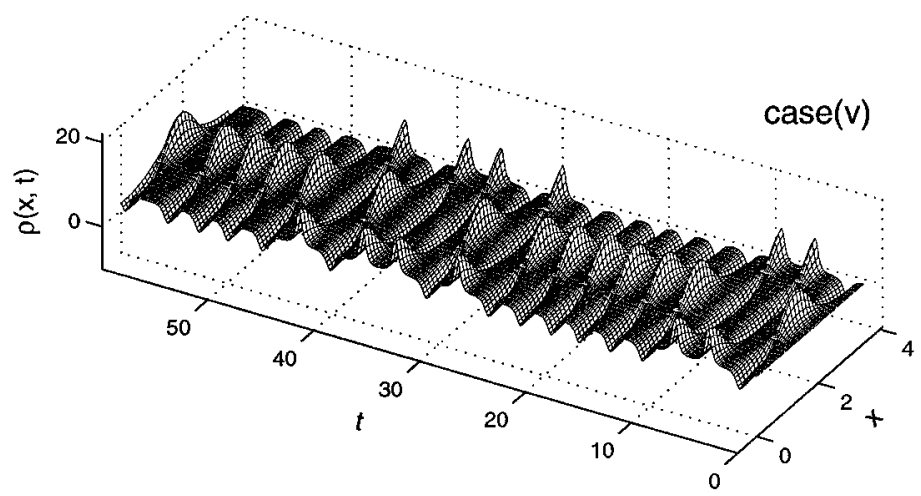

(a)

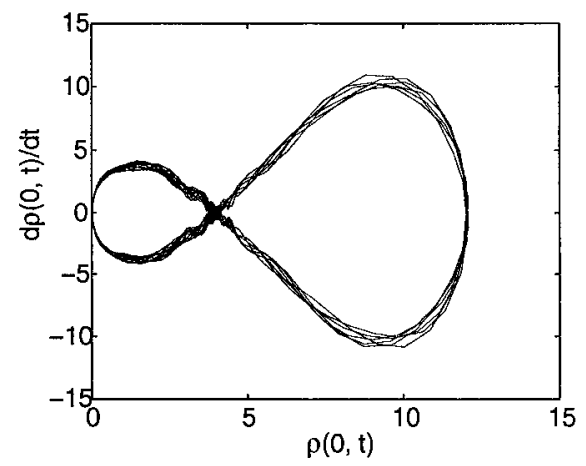

(b)

FIG. 3. Similar to Fig. 1 but for cases (v). (a) The square-root amplitude function. Note the pattern competition between types I and II. (b) Homoclinic structure in the phase space. Note that homoclinic crossings occur.

$$
\beta(t)=\frac{1-4 g \rho_{0}}{4 \rho_{0}} \int_{0}^{t} \alpha^{2}(t) d \tau
$$

Therefore the solution of Eq. (15) is

$$
\begin{gathered}
\rho_{2}=\frac{\alpha^{2}}{3 k_{0}^{2}}\left(\frac{3}{2}-4 g \rho_{0}\right) \cos (2 x), \\
\theta_{2}=\frac{1}{2 \rho_{0} k_{0}^{2}} \partial_{t} \alpha \cos (x) .
\end{gathered}
$$

For the third order in $\epsilon$, Eq. (12) gives

$$
\begin{aligned}
\partial_{x}^{2} \rho_{3}+\rho_{3}= & \frac{56 g \rho_{0}-80 g^{2} \rho_{0}^{2}-9}{6 k_{0}^{4}} \alpha^{3} \cos (3 x) \\
& +\frac{1}{k_{0}^{4}}\left[\partial_{t}^{2} a+\frac{3-16 g^{2} \rho_{0}^{2}}{6} \alpha^{3}+2 k_{0}^{3} k_{2} \alpha\right] \\
& \times \cos (x)+k_{0}^{2}\left(3-12 g \rho_{0}\right) \alpha^{2} \omega_{2}^{\prime}, \\
\partial_{x}^{2} \theta_{3}= & \frac{1}{k_{0}^{2}}\left[\frac{4 g}{3 k_{0}^{2}}-\frac{1-k_{0}^{2}}{2 \rho_{0} k_{0}^{2}}\right] \partial_{t} \alpha \cos (2 x) .
\end{aligned}
$$

In the first equation, the conservation of the quasiparticles requires $\omega_{2}^{\prime}=0$, and the periodic boundary condition requires the coefficient of $\cos x$ also to be zero, i.e.,

$$
\partial_{t}^{2} \alpha+\frac{3-16 g^{2} \rho_{0}^{2}}{6} \alpha^{3}+2 k_{0}^{3} \delta k \alpha=0 .
$$

Here we have replaced $k_{2}$ by $\delta k=k-k_{0}$ since $k_{1}=0$. This is an equation for $\alpha(t)$. The solutions of Eq. (18) are

$$
\begin{gathered}
\rho_{3}=\frac{1}{48 k_{0}^{4}}\left(9+80 g^{2} \rho_{0}^{2}-56 g \rho_{0}\right) \alpha^{3} \cos (3 x), \\
\theta_{3}=\frac{1}{2 k_{0}^{2}}\left[\frac{1-k_{0}^{2}}{2 \rho_{0} k_{0}^{2}}-\frac{4 g}{3 k_{0}^{2}}\right] \partial_{t} \alpha^{2} \cos (2 x) .
\end{gathered}
$$

Therefore the solutions of Eq. (12) up to the third order in $\epsilon$ are, after changing the variables $(x, t)$ back to $\left(k x, \omega^{\prime} t\right)$ and setting $\epsilon=1$,

$$
\begin{aligned}
\rho(x, t)= & \rho_{0}+\alpha(t) \cos (k x)+\frac{1}{3 k_{0}^{2}}\left(\frac{3}{2}-4 g \rho_{0}\right) \alpha^{2}(t) \cos (2 k x) \\
& +\frac{1}{48 k_{0}^{4}}\left(9+80 g^{2} \rho_{0}^{2}-56 g \rho_{0}\right) \alpha^{3}(t) \cos (3 k x), \\
\theta(x, t)= & \frac{1-4 g \rho_{0}}{4 \rho_{0}} \int_{0}^{t} \alpha^{2}(t) d \tau+\frac{1}{2 \rho_{0} k_{0}^{2}} \partial_{t} \alpha(t) \cos (k x) \\
& +\frac{1}{2 k_{0}^{2}}\left[\frac{1-k_{0}^{2}}{2 \rho_{0} k_{0}^{2}}-\frac{4 g}{3 k_{0}^{2}}\right] \partial_{t} \alpha^{2}(t) \cos (2 k x),
\end{aligned}
$$

where $\alpha(t)$ satisfies Eq. (19). Note that $\omega^{\prime}=1$ up to the 


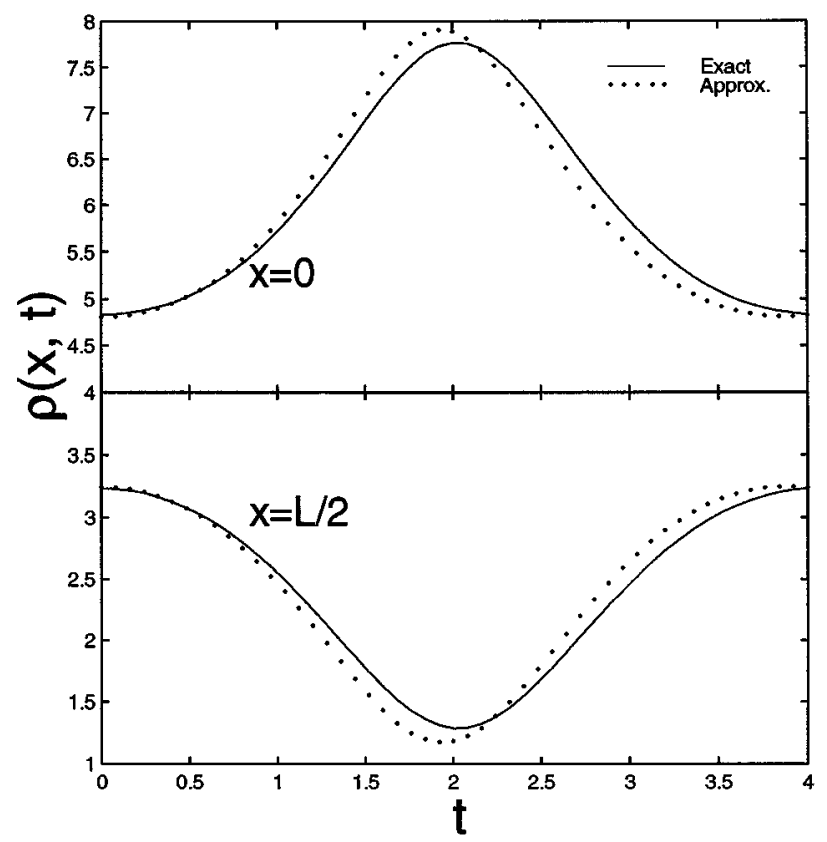

FIG. 4. Comparison between the approximation given by the perturbation analysis and the numerically determined exact solution of the initial-boundary value problem. The square-root amplitudes at $x=0$ and $x=L / 2$ are plotted. The exact (approximate) solution is given by the solid (dotted) curves.

second order in $\epsilon$ because of $\omega_{2}^{\prime}=0$ and that the form of Eq. (19) remains unchanged even after changing the time variable $t$ back to $\left(\omega^{\prime} t\right)$.

To see how good the approximation formula (21) is, we compare it with the numerically determined exact solution of the initial-boundary value problem. The numerical comparison is done for the GNS equation (2) where $E_{0}=2, g$ $=0.05$, and $L=2 \pi /\left(0.95 k_{0}\right)=2 \pi /\left[0.95 \sqrt{2 E_{0}^{2}\left(1-2 g E_{0}^{2}\right)}\right]$, with periodic boundary condition and with the initial conditions implicitly given by $\alpha(t=0)=0.8$ and $\partial_{t} \alpha(t=0)=0$. In determining the exact solution, we first substitute the initial values of $\alpha$ and $\partial_{t} \alpha$ into Eq. (21) to find the initial condition $E(x, 0)=\sqrt{\rho(x, 0)} e^{i \theta(x, 0)}$ for the GNS equation. With this initial condition and the periodic boundary condition, the GNS equation (2) is then numerically integrated in the same way as we did in Sec. II. The results are shown as the solid curves in Fig. 4. For the approximate solution, we first numerically solve the initial value problem for $\alpha(t)$, i.e., Eq. (19) with the initial conditions $\alpha(t=0)=0.8$ and $\partial_{t} \alpha(t=0)=0$. Using Eq. (21), we then obtain the approximate solution shown as the dotted curves in Fig. 4.

In Fig. 4, we plot the exact and the approximate squareroot amplitude $\rho(x, t)$ at two points in space, $x=0$ and $x$ $=L / 2$. It can be seen from the figure that the perturbation analysis gives a good approximation to the exact solution.

\section{PATTERN COMPETITION AND HOMOCLINIC STRUCTURE}

Let us consider a spatial shift by $L / 2$ in the initial condition of Eq. (9). The shift causes a switch between cases (i) and (ii) of the initial condition, that is, a switch between pattern types I and II,

$$
\rho^{(\mathrm{I})}\left(x+\frac{L}{2}, t=0\right)=\rho^{(\mathrm{II})}(x, t=0),
$$

where $\rho$ is decorated by pattern type. This relation holds for later times because the equation of motion and the boundary condition are both invariant under a translation in space. Therefore

$$
\rho^{(\mathrm{I})}\left(x+\frac{L}{2}, t\right)=\rho^{(\mathrm{II})}(x, t) .
$$

On the other hand, it is clear from Eq. (21) that the time dependence of $\rho(x, t)$ is solely on the order function $\alpha(t)$. Therefore we define $\bar{\rho}^{(\mathrm{II})}(x, \alpha(t))=\rho^{(\mathrm{I})}(x, t)$ and $\bar{\rho}^{(\mathrm{II})}(x, \alpha(t))=\rho^{(\mathrm{III})}(x, t)$. From Eqs. (21) and (23), we have

$$
\bar{\rho}^{-(\mathrm{I})}(x,-\alpha(t))=\bar{\rho}^{-(\mathrm{II})}(x, \alpha(t)) .
$$

This relation indicates that it is the sign of $\alpha(t)$ that determines which type of pattern will appear. Whenever $\alpha(t)$ changes its sign, the pattern type changes. If $\alpha(t)$ changes sign randomly, pattern alternates between types I and II randomly, and pattern competition occurs.

$\alpha(t)$ is given by the differential equation (19), which can be considered as the equation of motion for a Hamiltonian system whose Hamiltonian function is

$$
H(\alpha, p)=\frac{p^{2}}{2}+V(\alpha), \quad V(\alpha)=\frac{3-16 g^{2} \rho_{0}^{2}}{24} \alpha^{4}+k_{0}^{3} \delta k \alpha^{2},
$$

where $p=d \alpha / d t$. When $\delta k>0$, the potential $V(\alpha)$ is for a single well. Note that we only consider the case for $g$ small so that $g<\sqrt{3} /\left(4 E_{0}^{2}\right)$. When $\delta k<0$, however, the potential is a double-well potential. Hence $\delta k=0$ (i.e., $k=k_{0}$ ) is a critical point. This critical value $k_{0}$ has been previously obtained from the linear stability analysis shown in Sec. II. This is the threshold of modulation instability.

When $\delta k<0$, the double-well potential has a local maximum at $\alpha=0$, a well in the region $\alpha>0$, and another well in $\alpha<0$. In the phase space (the $\alpha p$ plane), this implies that there is a homoclinic orbit with two islands jointed at the origin, one island in the region $\alpha>0$ and the other in $\alpha$ $<0$. Since the sign of $\alpha(t)$ determines the type of pattern for our original system (described by the GNS equation (2) with the periodic boundary condition), the original system has a homoclinic structure.

The Hamiltonian of Eq. (25), obtained in the perturbation analysis up to the third order in $\epsilon$, represents an integrable Hamiltonian system. Performing the perturbation analysis to a sufficiently higher order, however, Eq. (25) with the higher-order terms should represent a nearly integrable Hamiltonian system because the original GNS equation (2) is nearly integrable. There is a thin disorder layer in the neighborhood of the homoclinic orbit for the system described by the GNS equation. The motion in this disorder layer causes random homoclinic crossings. Homoclinic crossing implies alternating between the two wells of the double-well potential, sign changing in $\alpha(t)$, and therefore switching between the two pattern types. 
From analysis presented above, it is clear that homoclinic crossings are the underlying mechanism for pattern competition in the system described by the GNS equation with the periodic boundary condition. Similar analysis can be done for other systems.

\section{CONCLUSION}

Artificial disturbances applied to the steady-state solution of the equation of motion can be used to select desirable patterns or to achieve pattern competition in a numerical simulation. The simulation for the system described by the GNS equation (1) reveals the correspondence between pattern competition and homoclinic crossing.

The perturbation analysis in the neighborhood of the steady-state solution of the GNS equation near the threshold of the modulation instability gives the equation for the order function $\alpha(t)$. Based on this equation, existence of homoclinic structure of the system can be shown and the correspondence between homoclinic crossing and pattern competition can be understood. The homoclinic crossings in time series are the mechanism of pattern competition.

Although this study is restricted to the GNS equation with the periodic boundary condition, we believe that the method used here can be applied to more general physical situations and similar conclusions are expected.

\section{ACKNOWLEDGMENTS}

This work was partially supported by the Research Grant Committee of Hong Kong under Grant Nos. HKUST606/95P and DAG94/95.SC01.
[1] D. C. Leslie, Developments in the Theory of Turbulence (Clarendon Press, Oxford, 1973).

[2] Y. S. Kivshar and B. A. Malomed, Rev. Mod. Phys. 61, 763 (1989), and references therein.

[3] M. C. Cross and D. C. Hohenberg, Rev. Mod. Phys. 65, 851 (1993), and references therein.

[4] F. T. Arecchi, Physica D 86, 297 (1995).

[5] J. M. Greene and J.-S. Kim, Physica D 33, 99 (1988).
[6] L. A. Kalyakin, Physica D 87, 193 (1995).

[7] H. C. Yuen, Phys. Fluids 21, 1275 (1978).

[8] H. T. Moon, Phys. Rev. Lett. 64, 412 (1990).

[9] C. T. Zhou, X. T. He, and S. G. Chen, Phys. Rev. A 46, 2277 (1992).

[10] E. Fermi, J. Pasta, and S. Ulam, Los-Alamos Scientific Report No. LA-1940, 1955. 